\title{
Editorial
}

\section{Curar y enfermar: apuntes sobre la filosofía de la enfermedad}

Alicia Natali Chamorro Muñoz

Universidad Industrial de Santander, Colombia 


\section{Curar y enfermar: apuntes sobre la filosofía de la enfermedad}

\section{Healing and Becoming: remarks on Philosophy of Sickness}

Forma de citar (APA): Chamorro Muñoz, A. N. (2020). Curar y enfermar: apuntes sobre la filosofía de la enfermedad. Revista Filosofía UIS, 19(2), https://doi. org/10.18273/revfil.v19n2-2020001

Forma de citar (Harvard): Chamorro Muñoz, A. N. (2020). Curar y enfermar: apuntes sobre la filosofía de la enfermedad. Revista Filosofía UIS, 19(2), 11-20.

Fecha de recepción: 12 de abril de 2020

Fecha de aceptación: 20 de abril de 2020

Alicia Natali Chamorro Muñoz: colombiana. Doctora en Filosofía. Profesora titular de la Escuela de Filosofía en la Universidad Industrial de Santander.

ORCID iD: https://orcid.org/0000-0002-7189-5943

Correo electrónico: anchamom@gmail.com 


\section{Curar y enfermar: apuntes sobre la filosofía de la enfermedad}

La filosofía ha pensado tradicionalmente los dilemas a los que nos enfrenta la muerte; incluso, se ha visto a sí misma como una preparación para la muerte y parece que su origen siempre se relaciona con el abismo de pensar la propia finitud. Otro tema, cercano y a la vez distinto, es la enfermedad a la cual la filosofía no ha dedicado tantas líneas; aun así, es un tema que merece nuestra reflexión como forma de enfrentarnos a la búsqueda de sentido que encierra para la creatura humana el dolor de la herida, la supuración de la llaga y el nerviosismo del no saber la gravedad de la dolencia. Para poder asumir lo anterior veremos los siguientes puntos: la relación entre vulnerabilidad y enfermedad; la frontera porosa entre ser sanos y ser enfermos; finalmente, la enfermedad y las relaciones interpersonales a las que ella apela.

\section{Vulnerabilidad humana y enfermedad}

Sin duda, nuestra existencia se define por la contingencia. Podemos incluso afirmar que somos casi siempre nuestras vulnerabilidades y pocas veces nuestra autonomía (Blumenberg, 2014). Incluso, la estructuración bípeda de nuestro cuerpo es ya un signo de la fragilidad que nos constituye, pues el bipedismo implica dificultades para mantener a nuestras crías y un arriesgarnos a que en nuestro deseo de ver cada vez más allá estemos aún más expuestos. Para el filósofo alemán Hans Blumenberg nuestra espalda es el símbolo de nuestra vulnerabilidad en cuanto puede ser vista desde diferentes frentes y atacada, ella es el punto no solo al que los otros pueden acceder sin que nosotros los veamos, sino que también su visibilidad nos es negada a nosotros mismos. Así, la espalda es la mejor metáfora de nuestra indisponibilidad y de la imposibilidad de una imagen transparente de nosotros mismos: "La visibilidad del cuerpo propio es una experiencia opaca por naturaleza para la conciencia, una experiencia que paradójicamente sólo conoce por los otros" (Blumenberg, 2014, p. 152). En este mismo camino, la natalidad y la mortalidad son eventos que suceden en comunidad, con otros, y lo humano está determinado por la interacción de estos otros que nos acogen y determinan; así, nuestra individualidad está mediada por la condición de ser frágiles y necesitados de comunidad (Marquard, 2000, p. 43).

Ahora bien, normalmente la vulnerabilidad se define como la pérdida del poder ante otro. Por ejemplo, cuando hablamos de la vulnerabilidad de un país al no poder mantener las fronteras protegidas de cualquier invasión, un espacio 
vulnerable implica que puede ser atravesado por lo externo, ser conquistado por lo otro al ser débil (Have, 2016). Desde esta perspectiva un problema de vulnerabilidad es, entonces, un problema de seguridad, que encontraría su solución en la reconstrucción de fronteras y en la posibilidad de erradicación de lo que constituyó en un primer momento el estado de debilidad. Este sentido de vulnerabilidad se ha colado en la comprensión de la enfermedad. Ser vulnerables implica que tenemos siempre un flanco débil que está a disposición de ser dañado por un agente patógeno, un gen mal replicado o un virus. La corporalidad nos convierte en seres de fronteras porosas que pueden ser dañadas y ultrajadas.

Empero, es posible llevar a un nivel de mayor profundidad la idea inicial de vulnerabilidad a partir de comprenderla en su dimensión existencial (Fuchs, 2015). Hablar de una vulnerabilidad existencial implica comprender que somos creaturas sensibles y precarias, necesitados de ser acogidos por los otros antes de cualquier determinación individual, como lo señala la filósofa Judith Butler (2006) la condición humana es la de una vida precaria, que por su fragilidad constitutiva necesita de cuidado y acogida. Entonces, la vulnerabilidad no es simplemente una falla o vacío que debe ser superado a partir de generar algunos tipos de fortificaciones en las partes débiles de nuestros cuerpos, antes bien la vulnerabilidad nos constituye e incluso permite la apertura a una constitución personal y a una vida que vale la pena ser vivida. Esta doble cara de la vulnerabilidad es resaltada por Corine Pelluchon (2013) en su lectura de la alteridad: "la proximidad es la exposición al otro, es decir, que la subjetividad es aprehendida en su alteridad, la del otro, no sintetizada ni reducida a lo mismo, afectada por el otro, la alteridad en sí que hace posible la escucha, la proximidad y la compasión" (p. 230). Por ejemplo, en condiciones normales la técnica ha permitido la generación de un mundo posible y adecuado, a partir de convertir los peligros que se presentan en el horizonte dentro de un umbral de lo cómodo; aun así, la comodidad ofrecida por la técnica no puede erradicar la vulnerabilidad existencial. Esto sucede así, porque en la salida del ser humano de la selección meramente darwinista hacia el universo cultural lo que en verdad se presenta es el paso de depender de unos aspectos a otros. Por tanto, algo así como una erradicación absoluta de la vulnerabilidad absoluta, en cuanto eliminación de todos los peligros, es realmente algo imposible para el ser humano y se constituye en signo necesario de su sobrevivencia como especie (Blumenberg, 2014).

Ahora bien, pese a que todos somos frágiles y dependientes, tales condiciones de vulnerabilidad pueden variar a lo largo de la historia personal vital y se determinan por todo tipo de escenarios, entre ellos los políticos y los económicos. Nuestra vulnerabilidad puede manifestarse episódicamente más patente, fundamentalmente en la enfermedad. Tampoco todo enfermo es igual de vulnerable ni recibe los mismos cuidados. 
La comprensión de la vulnerabilidad que acaece cuando estamos enfermos nos sitúa no solo frente a la dimensión de la finitud, sino también ante la reflexión sobre el cuidado. Ante la enfermedad la filosofía se enfrenta al dolor y a la fragilidad de estar entregados completamente al otro (Schumacher, 2010). Esta comprensión filosófica de la vulnerabilidad que se presenta en el cuerpo del enfermo - que muestra su herida, del que sus fronteras se han vencido y que apela por un cuidado - redefine la comprensión que podemos tener del sentido de la vida en conexión con nuestra composición biológica: "Esta co-presencia en el sufrimiento del prójimo, indispensable para cambiar la mirada y hacer efectiva la solidaridad, nos remite a la vulnerabilidad constitutiva de los humanos que somos, en el cruce de la biología y el sentido" (Kristeva, 2009, p. 142). En los momentos de enfermedad, donde nos convertimos en fragilidad patente ante el otro, parece que no es tan claro que somos el ser-para-la-muerte - a la que se refería Heidegger - ; antes bien, la creatura humana se presenta como un ser para la vida que requiere cuidados (Pelluchon, 2013). En este contexto, la comprensión clásica de la Sorge se redefine a través de la comprensión de lo vulnerable.

Esta caracterización de la vulnerabilidad en la experiencia de la enfermedad se puede comprender desde dos dimensiones: como cuerpo vivo (Leib) y como cuerpo físico (Körper). Siguiendo a Plessner, podemos señalar que nosotros tenemos un cuerpo físico (Körper) y somos un cuerpo vivo (Leib) (Plessner, 1975). Tener un cuerpo físico significa que asumimos la corporalidad como algo externo, un instrumento para su uso expuesto como materialidad ante la ciencia o como un objeto que se puede localizar y poseer. Por su parte, el ser un cuerpo vivo (sein Leib) asume nuestra condición de ser corpóreos. Se resalta la unidad con este cuerpo que somos en cada momento, desde el cual vivimos el mundo. La relación entre el cuerpo-físico y el cuerpo-vivo no es del todo clara, aunque la podemos diferenciar conceptualmente; podemos asumirla más bien como un lugar de tránsito que procura describir nuestra experiencia en el mundo como seres que tienen una conciencia personal de su propio cuerpo. Incluso, podemos tener la experiencia de la polaridad y la tensión de estas dos dimensiones al mismo tiempo. Esto sucede de manera particular en el caso de la enfermedad, en donde se da de manera más patente esa semipertenencia con el cuerpo mismo.

\section{2. ¿Qué significa ser sano o estar enfermo?}

El concepto de salud no puede ser comprendido sin la consideración de la enfermedad, pues ambos parecen siempre estar presentes en la condición humana. Parece que estar saludable se parece a un estado en donde no caemos "en cuenta" de la propia corporalidad finita. Vivir en un estado de salud es vivir como si no tuviera que caer en cuenta que tengo un cuerpo, en tanto este se 
presenta como transparente durante mi experiencia con el mundo (Blumenberg, 2014, p. 523).

Entonces, el paso de un estado de salud a uno de enfermedad es generalmente de transición, que va de un casi no notar el cuerpo a asumir que el cuidado del cuerpo es el centro del día y, lamentablemente para muchos también, de la noche. El cuerpo como cuerpo sano es un cuerpo transparente, que no se siente; por esta razón, Canguilhem (1971) sigue a Lerich y apela a la metáfora del "silencio de los órganos" (p. 46), para indicar nuestra experiencia de la salud. En ella tenemos la sensación de una comunicación directa entre nuestra experiencia del mundo y nuestro cuerpo vivo, permitiéndonos olvidar el funcionamiento de cada uno de los órganos que lo componen. Igualmente, la experiencia de la enfermedad hace al hombre consciente de su propio cuerpo. Aquí experimenta su propia corporalidad no como un medio, sino que el cuerpo se convierte en algo que ata, que impide, que tiene algo externo a sí mismo y que debe, por tanto, superar o vencer. Aunque se tenga una parte enferma del cuerpo o esté dañada, el organismo entero es realmente el que está enfermo. Así, la pregunta que se nos presenta es: ¿qué es salir de pasar desapercibido para sí mismo?

Por una parte, es claro que estos estados de transición deben superar la idealidad de un humano completamente saludable, como afirma Von Weizsäcker. La idea de que siempre estamos sanos y la enfermedad es algo que acaece de manera transitoria es lamentablemente falsa, pues en la vida normal de un ser vivo concurren continuamente estados patológicos y, por ende, establecer lo normal a diferencia de la patológico es menos simple de lo esperado (Weizsäcker, 2005, pp. 17-18). Entonces los procesos de salud y de enfermedad son de umbrales y la capacidad de pasar de un umbral al otro depende de diversas condiciones particulares del individuo. Entender la enfermedad como una cuestión de umbrales permite comprender de mejor manera esta experiencia existencial, donde enfermedad y salud no son entes ontológicamente diferentes "disputándose el cuerpo del paciente", sino que la diferencia se determina por una desproporción y la enfermedad surge desde condiciones propias dentro del sistema (Canguilhem, 1971).

Sería necesario pensar algo así como que la enfermedad hace parte del organismo vivo, pues es un acontecimiento posible dentro de sus funciones. Pero, es necesario asumir al mismo tiempo que es un acontecimiento novedoso y retador. Entonces, que el síntoma patológico visto aisladamente pueda ser explicado como un aumento o disminución de lo que podría ser una función normal, no excluye que, a nivel del conjunto, como organismo en su totalidad, la vida en la enfermedad se experimente como otra forma de vida. Al respecto afirma Canguilhem (1971): 
¿no convendría decir, al fin de cuentas, que el hecho patológico sólo es captable como tal, es decir, como alteración del estado normal, en el nivel de la totalidad orgánica y, tratándose del hombre, en el nivel de la totalidad individual consciente donde la enfermedad se convierte en una especie de mal? (p. 60)

Podríamos considerar que el cuerpo del gravemente enfermo se le convierte al individuo en todo el contenido de su vida, en el motivo de angustia y a la vez de cuidado. El cuerpo se convierte en un objeto, que ya no es el cuerpo propio privadísimo, sino sometido a la intersubjetividad y necesitado de cuidados. El enfermo se puede equivocar a veces frente a sus propias dolencias; sin embargo, eso no invalida su genuino sentirse distinto y desde este último punto, la enfermedad podría pensarse como algo distinto al mero sufrir síntomas en determinados órganos; es decir, aunque todos enfermemos, la enfermedad es una experiencia originaria y original para cada uno de nosotros. La enfermedad es una experiencia factual de todo el organismo, aunque las dolencias estén situadas en un órgano o parte específica del cuerpo. De esta manera, esta es una situación límite en la que se experimenta un nivel mayor de vulnerabilidad porque la corporalidad está expuesta y necesitada de los otros.

En este sentido, la vulnerabilidad propia de la enfermedad oscila entre la percepción de nuestra corporalidad como cuerpo físico - que está frente al servicio médico y del cual el sujeto se siente lejano o abstraído- a su propia comprensión como cuerpo vivo - ese yo qué está enfermo y el cual trata de sobrevivir-. En esta última comprensión el enfermo asume la preocupación de sí y con ello surgen nuevas inquietudes sobre el modo como debe continuar viviendo y cómo reconfigurar su identidad pese a su enfermedad. Como corolario para reflexionar nos queda el siguiente: Si en la experiencia de la enfermedad el individuo está afectado en su totalidad, su tratamiento debería involucrar, de una u otra manera, igualmente esta totalidad.

\section{La relación con los otros y la incomunicabilidad de la enfermedad}

Estamos ante la paradoja de que todos nos enfermamos, pero cada uno vive su enfermedad de una forma individual y particular en tanto experiencia propia. Esto se muestra en la herida como un símbolo que vincula la manifestación de soledad e incomunicabilidad del dolor con la necesidad de cuidado y atención; al sufrir una enfermedad estamos atravesados por la experiencia de la fragilidad profunda. Señala Canguilhem (1971) en Lo normal y lo patológico:

La enfermedad es conmoción y puesta en peligro de la existencia. Por consiguiente, la definición de la enfermedad requiere como punto de 
partida la noción de ser individual. La enfermedad aparece cuando el organismo es modificado de manera tal que llega a reacciones catastróficas dentro del medio ambiente que le es propio. (p. 140)

La enfermedad es una experiencia significativa que transforma de manera decisiva las formas de relacionarse. Cuando el hombre está enfermo se encuentra en un estado de conmoción entre lo que su organismo puede hacer y aquello que el medio le exige. La enfermedad es, entonces, la experiencia de sentir la catástrofe, aquello no esperado, como ruptura del individuo con el medio que le es propio. Igualmente, quien está enfermo necesita a la vez compensar sus carencias. Por esta razón, busca organizar nuevas formas de interacción, que le permitan continuar viviendo, a pesar de la vulnerabilidad a la que está expuesto.

En este orden de ideas, el mayor grado de esta catástrofe es la extrañeza de sí mismo. La corporalidad, que hemos descrito como cuerpo vivo, se vuelve extraña para el enfermo, que se siente a veces un extranjero en su propia corporalidad, en cuanto la enfermedad cierra posibilidades que anteriormente se tenían abiertas en el estado de salud (Canguilhem, 1971, p. 151). En este sentido, la diferencia entre un organismo sano y otro enfermo radica en que este último no tiene las formas habituales de poder responder a su medio y asegurarse una respuesta adecuada para el futuro. En cierta forma pierde aquello que era su mundo cotidiano, incluso en los grados de mayor vulnerabilidad las tareas más simples del organismo son sobre-exigencias para el enfermo.

El cuerpo del hombre enfermo se presenta como una semi-transparecia para los otros; por una parte, este cuerpo está expuesto, en su fragilidad, a la interacción con el mundo y con su tejido social, permitiendo así su lectura; es decir, el enfermo no está aislado completamente del mundo, sino que interactúa desde sus posibilidades y limitaciones. Por la otra, también es algo que siempre se oculta, ya que no es posible descifrarlo de manera absoluta; el sufrimiento del enfermo queda vedado para los otros, en la medida en que toda lectura es imperfecta. Esta perspectiva de la semi-transparencia de la enfermedad para los otros nos permite apartarnos también de la visión de la enfermedad como una experiencia de afección externa; esto es, entender la enfermedad como un algo absolutamente externo que le acaece al sujeto. El problema con esta visión radica en que escinde al enfermo de su experiencia vital, a la vez que determina el camino de la curación como una guerra y conduce a la pregunta sobre la causa del estar enfermo entendida como un castigo (Frick, 2000). La búsqueda de un culpable en relación con la enfermedad conduce a la imposibilidad de comprensión del enfermo, e incluso a culparlo de su propio mal. Esta perspectiva que comprende la causa de la enfermedad como algo completamente externo ha sido también explorada por Mukherjee (2012) y Sontag (1978) en sus estudios sobre el cáncer. Ambos autores coinciden en denunciar los usos de lenguaje en los cuales la enfermedad se asume como algo externo, ya que estos usos convierten 
peligrosamente al enfermo en un campo de batalla; dicho con otras palabras, el cuerpo es un espacio para la erradicación de un enemigo. Estos esfuerzos de eliminación pueden implicar la deshumanización del tratamiento.

Ahora bien, frente a la enfermedad nos embarga el miedo, inclusive casi más que la angustia, ya que todos estamos en un frágil equilibrio entre perdernos en la extrema preocupación sobre nuestro cuerpo o la despreocupación absoluta de dejarnos a los otros. Frente a esta doble ciudadanía del reino de lo sano y de lo enfermo, como lo menciona Sontag (1974), el humano se proyecta como un ser para y por el cuidado expuesto a los otros, en este caso se presentan preguntas fundamentales como: ¿cuáles son las dicotomías que se presentan en el sí mismo en relación con el sufrimiento que se intenta manifestar y los otros comprenden? y żcómo el sufrimiento se maneja dentro de un sistema cultural de construcción identitaria?

Para terminar, no podemos dejar de anotar el paso que implica pensar la enfermedad ahora como pandemia. Como hemos visto, la vulnerabilidad existencial nos conecta y nos convierte en seres dependientes los unos de los otros; asimismo, el sufrimiento de la enfermedad tiene un alto grado de incomunicabilidad, pues nadie puede hacer absolutamente transparente su dolor, ni tampoco nadie puede sentir como tal el dolor del otro. Aun así, como vimos, la enfermedad nos conecta, como seres vulnerables, en nuestros proyectos de felicidad en comunidad, fundamentalmente con el sentido humano que se puede perder, pues con cada muerte se empobrece el mundo y con cada nacimiento se enriquece. Una pandemia muestra de manera radical la conexión profunda que tenemos los unos con los otros; pues estamos ante la posibilidad de enfermar colectivamente. A la vez que, se exacerba el miedo a todo contacto que nos puede enfermar, al espacio en el que antes nos movíamos. La pandemia nos muestra una nueva dimensión de la enfermedad: una enfermedad de todos, la enfermedad propia del mundo al que estábamos habituados.

\section{Referencias}

Blumenberg, H. (2014). Beschreibung des Menschen. Suhrkamp.

Butler, J. (2006). Vida precaria. El poder del duelo y la violencia. (F. Rodríguez, trad.). Paidós.

Canguilhem, G. (1971). Lo normal y lo patológico. (R. Potschart, trad.). Siglo XXI Editories.

Frick, E. (2000). Curar por la herida. Sobre el psicoanálisis del arquetipo de curación. (S. Mayer, trad.). Lumen. 
Fuchs, T. (2015). „Körper haben oder Leib sein“. Gesprächspsychotherapie und Personzentrierte, Beratung 3, (15). 147-153.

Have, L. (2016). Vulnerability. Challenging bioethics. Routledge.

Kristeva, J. (2009). Esa increíble necesidad de creer: un punto de vista laico. (V. Ackerman, S. Mattoni, trads.). Paidós.

Marquard, O. (2000). Philosophie des Stattdessen. Reclam.

Mukherjee, S. (2012). El emperador de todos los males. (H. Pons, trad.). Taurus.

Pelluchon, C. (2013). La autonomía quebrada. Bioética y filosofía. (A. Marín, trad.). Editorial Universidad el Bosque.

Pelluchon, C. (2015). Elementos para una ética de la vulnerabilidad. Los hombres, los animales, la naturaleza. (J. Mejía, trad.). Pontificia Universidad Javeriana.

Plessner, H. (1975). Die Stufen des Organischen un der Mensch. Einleitung in die philosophische Anthropologie. Walter de Gruyter.

Schumacher, L. (2010). Philosophie. En Wittwer H, Schäfer D, Frewer A (Ed.), Sterben und Tod. Geschichte Theorie Ethik. Ein interdisziplinäre Handbuch. (pp. 28-38) WBG.

Sontang, S. (1978). Illness as Metaphor. Farrar, Straus and Giroux.

Von Weizsäcker, V. (2005). Patosofía. (D. Busch, trad.). Libros del Zorzal. 